\title{
Ecoulements tridimensionnels supercavitants : traitement numérique en théorie exacte, expérimentation en tunnel hydrodynamique, analyse critique des résultats
}

\author{
Supercavitating three-dimensional flows. \\ Numerical treatment in exact theory. \\ Experiments in a hydrodynamic tunnel. \\ Critical analysis of the results
}

\author{
A.R. Laali, \\ Ch. Pellone* \\ Docteur-Ingénieur, Hydrodynamique \\ Attaché de Recherche au C.N.R.S.
}

Principales notations

$A \quad$ : allongement

$C \quad$ : corde du profil

$\left.C_{D}=F_{0} / 0,5 V_{\infty}^{2} S_{a}\right)$

$\left.C_{L}=F_{L} / 0,5 V_{\infty}^{2} S_{a}^{a}\right\}$ coefficients d'efforts

$C_{M}=F_{M} / 0,5 V_{\infty}^{2} S_{a} C$

$C_{p}=Q_{\text {air }} / \rho_{\text {air }} S_{h} \mathscr{V}_{\infty}$ : coefficient du débit d'air

$h_{1}:$ profondeur d'immersion

$K=\left(P_{o}-P_{c}+\rho g h_{1}\right) / 0,5 \rho V_{\infty}^{2}:$ nombre de ventilation

$l \quad:$ longueur de la cavité

$P_{o} \quad$ : pression surface libre

$P_{a} \quad$ : pression atmosphérique

$P_{c} \quad$ : pression totale dans la cavité

$P_{v}^{c} \quad$ : pression de vapeur saturante de l'eau

$Q_{\text {air }}$ : débit d'air injecté

$S_{a} \quad$ : surface en plan de l'aile

$S_{h} \quad$ : surface de la base de l'aile

$\alpha \quad$ : incidence

$\sigma=\left(P_{o}-P_{v}+\rho g h_{1}\right) / 0,5 \rho V_{\infty}^{2}:$ nombre de cavitation

$\rho \quad:$ masse volumique de l'eau

$\rho_{\text {air }}:$ masse volumique de l'air

$\theta$ : flèche

\section{Introduction}

Depuis une douzaine d'années, des études ont été développées à l'Institut de Mécanique de Grenoble, dans le domaine des ailes sous-marines. Les résultats obtenus ont permis d'approfondir et d'élargir les connaissances dans ce domaine, et d'envisager des applications pour la construction de navires à hautes performances, l'augmentation des possibilités d'utilisation d'organes de machines, le fonctionnement des organes

\footnotetext{
* Institut de Mécanique de Grenoble, B.P. 53 X, 38041 Grenoble Cedex.
}

hydrauliques dans des gammes de vitesses supérieures aux vitesses nominales [1] à [4], [7].

Jusqu'à présent, les travaux effectués ont surtout porté sur l'étude des écoulements bidimensionnels. Le problème tridimensionnel offre des difficultés supplémentaires tant sur le plan théorique qu'expérimental, et pour les aborder, une maîtrise assez complète du problème bidimensionnel est nécessaire.

Sur le plan expérimental, une investigation rigoureuse portant sur des ailes limitées en envergure, nécessite une installation de grandes dimensions pour minimiser l'influence des parois, les effets de couche limite, les effets d'encastrement, aussi bien que pour minimiser les erreurs dues à la définition géométrique de l'aile elle-même.

L'approche théorique du problème tridimensionnel est difficile en raison principalement de la présence de la cavité dont la forme est inconnue. La méthode intégrale résumée dans cette étude permet de traiter aussi bien l'écoulement bidimensionnel que l'écoulement tridimensionnel d'un fluide parfait et incompressible autour d'une aile supercavitante [5], [6], [8]. La méthode des singularités à répartition discrétisée conduit essentiellement à résoudre un système linéaire. Le nombre de ventilation étant fixé, le programme de calcul permet d'obtenir le coefficient de pression minimum sur l'aile en fonction du coefficient de portance (domaine de non cavitation). Dans chaque cas traité, le calcul de la forme exacte de la cavité a été réalisé à l'aide d'une procédure itérative qui permet d'obtenir celle-ci numériquement point par point.

Pour valider une méthode de calcul, aussi bien que pour acquérir des informations intéressantes concernant le fonctionnement des ailes tridimentionnelles, et notamment l'influence de la forme en plan sur le domaine de non-cavitation, il était nécessaire de mener une campagne de mesures sur un tunnel de grande capacité. Les résultats de cette campagne sont exposés dans cet article. Ils sont étayés par une étude théorique 
dont on donne une brève présentation et ils font l'objet d'une analyse critique qui concerne l'influence des principaux paramètres géométriques et le domaine de non cavitation.

Les essais ont été effectués sur le tunnel hydrodynamique TH2-TH8 de la Société NEYRTEC dont la plus petite veine (Section $0,45 \mathrm{~m} \times 0,60 \mathrm{~m}$ ) permet une investigation des écoulements tridimensionnels dans des conditions acceptables. La série d'essais dont il est rendu compte porte sur une aile correspondant à un profil immuable, dont on a fait varier la flèche ainsi que l'allongement, par rotation autour d'un axe situé à l'emplanture et par troncatures successives. La même aile a fait l'objet d'une série d'essais en écoulement bidimensionnel sur la même installation, afin de valider l'installation par comparaison avec les calculs théoriques précédemment effectués en écoulement bidimensionnel par A. Rowe et J.L. Kueny [7].

\section{Analyse expérimentale}

\subsection{Description expérimentale}

\subsubsection{Moyen d'essai}

Le moyen d'essai est le tunnel hydrodynamique TH2TH8 de la Société NEYRTEC (fig. 1). Ce tunnel est conçu de façon à permettre la réalisation d'essais sur des maquettes de grandes dimensions. Il possède deux branches : la première est montée directement dans le circuit et admet un débit maximal de l'ordre de $8 \mathrm{~m}^{3} / \mathrm{sec}$. La deuxième branche dans laquelle les essais ont été effectués est dérivée de la première ; elle admet un débit maximal de l'ordre de $2 \mathrm{~m}^{3} / \mathrm{sec}$. Les deux branches se

TUNNEL HYOROOYNAMIQUE $8 \mathrm{~m}^{3 / 5}$

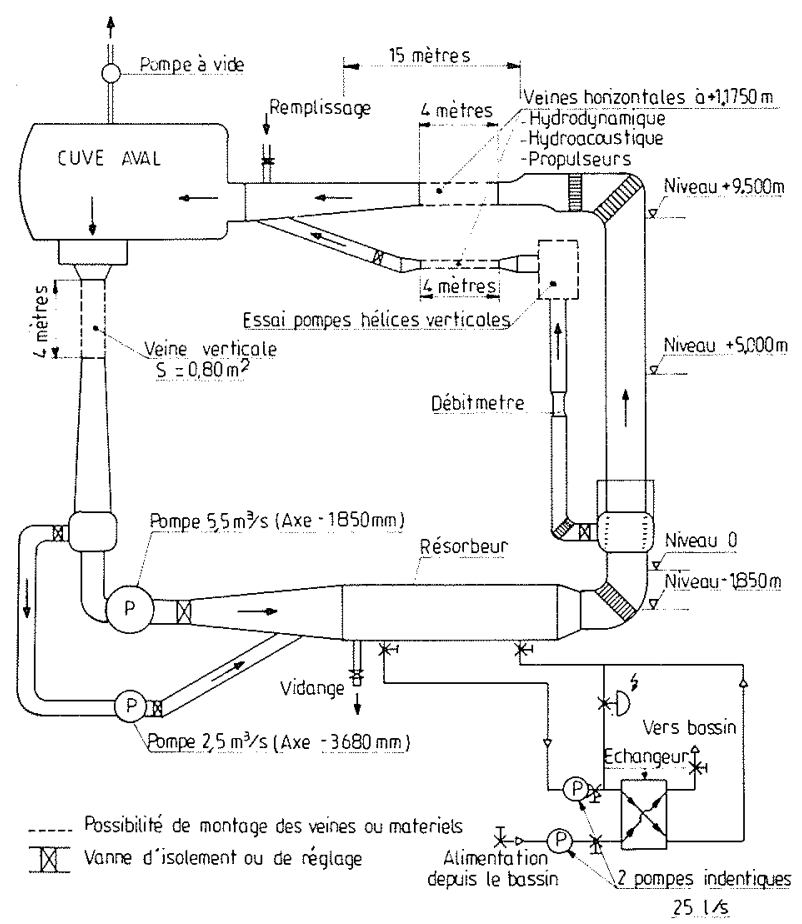

Figure 1 - Schéma du tunnel hydrodynamique $8 \mathrm{~m}^{3} / \mathrm{s}$ raccordent en aval à une cuve $\left(65 \mathrm{~m}^{3}\right.$ dont $50 \mathrm{~m}^{3}$ d'eau) à surface libre qui joue un rôle essentiel pour la fixation de la valeur de la teneur en air de l'eau.

Lorsqu'il s'agit d'essais à surface libre, une casquette amovible fixe la position de la surface libre. Dans le cas présent, cette surface était fixée à $450 \mathrm{~mm}$ du fond. La section d'essai a une largeur de $600 \mathrm{~mm}$, largeur tout à fait suffisante pour effectuer des essais tridimensionnels dans de bonnes conditions.

Pour les essais bidimensionnels, on sépare l'écoulement en deux parties en disposant à l'extrémité de l'aile une plaque plane parallèle aux parois latérales.

Un circuit d'air permet de pressuriser ou dépressuriser la surface libre. On peut atteindre ainsi une surpression de deux bars et une dépression de l'ordre de 0,97 bar au niveau de la surface libre. Cette différence de pression est supportée par la veine qui est blindée et sur laquelle on a prévu des fenêtres en plexiglas qui permettent la visualisation de l'écoulement.

\subsubsection{Méthode de mesure}

\section{Mesure des vitesses}

Le débit d'eau total dans le tunnel est mesuré à l'aide d'un débitmètre à hélice $\left(\varnothing 0,630 \mathrm{~m}\right.$ jusqu'à $\left.2 \mathrm{~m}^{3} / \mathrm{s}\right)$. Ce débitmètre à caractéristiques linéaires émet un signal sinusoïdal dont la fréquence est convertie en valeur de vitesse à partir du coefficient d'étalonnage qui tient compte de la section d'essais.

La vitesse de l'écoulement est fixée à $7,5 \mathrm{~m} / \mathrm{s}$ dans tous les essais. Cette vitesse est pratiquement la vitesse maximale qu'on peut obtenir avec le montage utilisé. $\mathrm{La}$ vitesse de l'écoulement, constante dans tous les essais, est suffisante pour qu'on puisse considérer comme négligeable une éventuelle influence du nombre de Froude sur les résultats.

\section{Mesure des pressions}

La pression dans la cavité, prélevée par l'intermédiaire d'une sonde cylindrique $(\phi \approx 2 \mathrm{~mm})$, encastrée à la base du profil, est renvoyée à l'une des branches d'un manomètre différentiel à eau. La pression de référence est celle de l'intérieur du caisson de sorte que l'on obtient directement la quantité $P_{o}-P_{c}$.

La précision attendue est de $\pm 1 \mathrm{~mm}$ d'eau lorsque la prise de pression n'est pas obturée par des goutelettes d'eau. Avant chaque mesure de la pression dans la cavité, il faut systématiquement injecter de l'air dans la sonde pour la déboucher.

La pression du caisson est mesurée par rapport à la pression atmosphèrique à $0,5 \mathrm{~mm}$ de mercure près; le baromètre donne une précision de l'ordre de $1 / 10$ de mm de mercure, toutes corrections de zéro effectuées.

\section{Mesure de la teneur en air de l'eau}

La mesure de la teneur en air de l'eau a été effectuée systématiquement pour les quatre demières configurations de l'aile étudiée, à l'aide d'un appareil Beckman.

La teneur en air dépend essentiellement de la pression et elle est une fonction directe de cette dernière dans la cuve aval du tunnel. Sur la deuxième branche du tunnel TH2-TH8, il existe une liaison directe entre la surface libre de la cuve aval et celle de la veine d'essais. Par conséquent, on ne peut pas régler la pression dans la cuve aval indépendamment de celle de la veine. Ainsi 


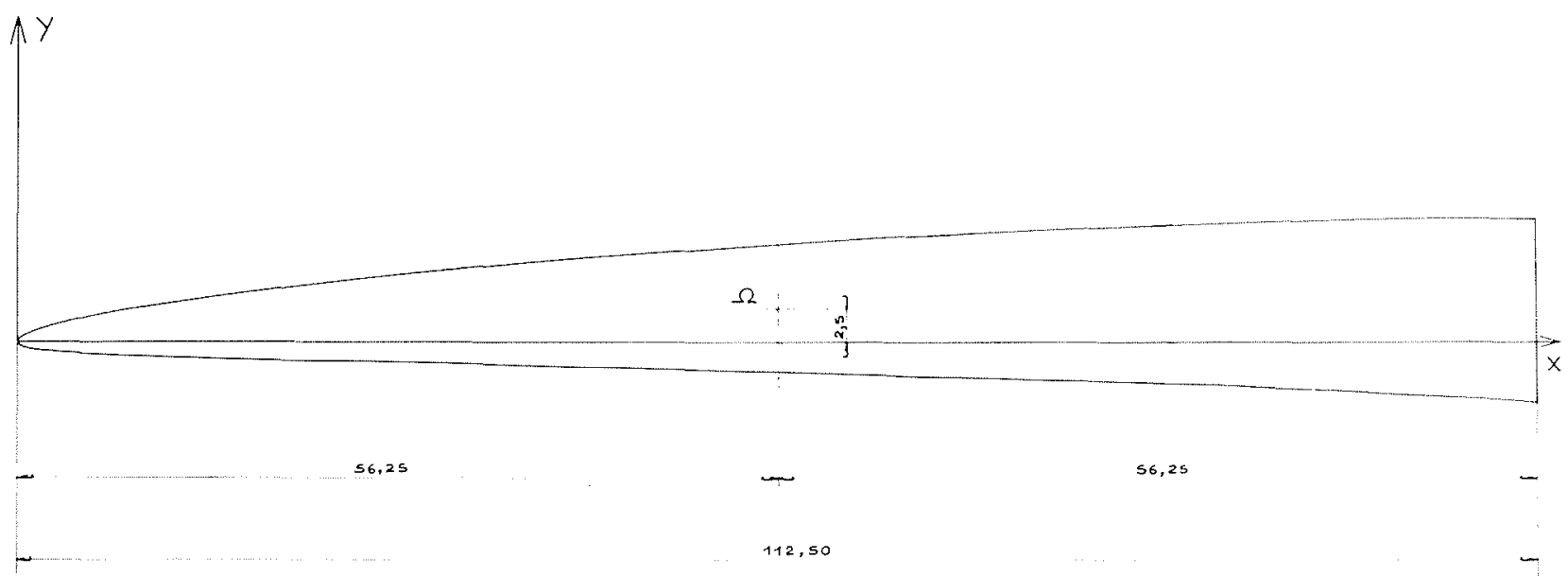

Figure 2 - Profil étudié.

la teneur en air de l'eau (qui est exprimée en pourcentage par rapport au pourcentage correspondant à la saturation) est fixée par les paramètres de l'écoulement.

Mesure des efforts hydrodynamiques - Balance La balance utilisée pour mesurer les efforts est une balance de paroi à trois voies. Elle est équipée de trois ponts complets de jauges de contrainte. Deux ponts donnent les efforts dans deux plans perpendiculaires en fonction de la flexion du barreau $\left(F_{x}\right.$ et $F_{z}$ ). Le troisième pont donne le moment suivant l'autre axe en fonction de la torsion du barreau. La capacité de la balance exprimée en moments correspond aux valeurs maximales suivantes : portance et traînée : 225 N.m; moment : 270 N.m.

Acquisition des données et chaîne de mesure

Les signaux émis par les ponts de jauges sont introduits directement dans un micro-processeur. Le dépouillement des mesures est entièrement automatisé par un programme de calcul sur micro-ordinateur.

Les signaux pris en compte au moment de l'acquisition des données sont corrigés en fonction des valeurs

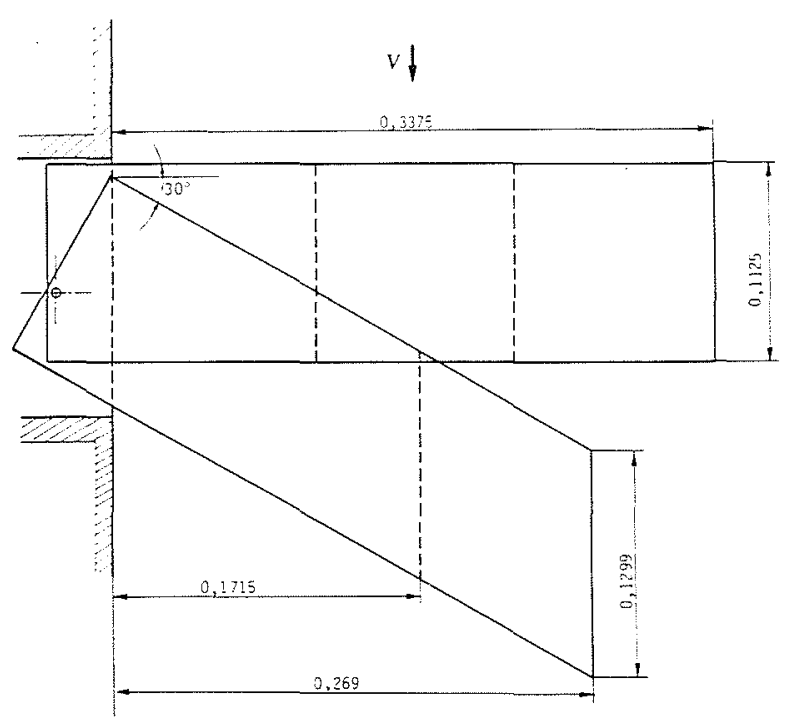

Figure 3 - Formés en plan de l'aile de référence obtenues à action hydrodynamique faible ; ces valeurs sont mémorisées après étalonnage.

\subsubsection{Description de l'aile étudiée}

Nous avons étudié le profil à coefficient de pression constant défini précédemment par Rowe [9] (fig. 2) correspondant à la répartition de pression symétrique $C_{p i}=-C_{p e}=0,12$; le calcul du profil tient compte de l'effet de surface libre (profondeur d'immersion : 1 ; nombre de ventilation nul). L'épaisseur relative de ce profil est de $12 \%$, ce qui assure à la fois une résistance mécanique convenable et une faible valeur du coefficient de traînée. Le rayon de courbure au bord d'attaque est sensiblement égal à $0,0025 \mathrm{C}$, soit $0,2 \mathrm{~mm}$.

La surface du modèle bidimensionnel est égale à $112,5 \times 337,5 \mathrm{~mm}^{2}$. Après avoir effectué les essais en écoulement bidimensionnel, le profil a été tronqué pour qu'on dispose à chaque fois d'une nouvelle configuration tridimensionnelle (fig. 3). Le tableau I rassemble les caractéristiques des différentes géométries qu'on a obtenues en tronquant le modèle original. Ces caractéristiques sont :

$\begin{array}{ll}\text { Flèche } & : \theta \\ \text { Allongement } & : A \\ \text { Surface en plan de l'aile } & : S_{a} \\ \text { Surface de la base de l'aile } & : S_{m} \\ \text { Envergure de l'aile } & : L \\ \text { Corde } & : C\end{array}$

La figure 3 montre la géométrie de l'aile en flèche.

\begin{tabular}{|c|c|c|c|c|c|c|}
\hline \multicolumn{7}{|c|}{ Tableau I } \\
\hline $\begin{array}{c}\text { Conflgura- } \\
\text { tlon } \\
N^{\circ}\end{array}$ & $\theta^{\circ}$ & $A$ & $\begin{array}{c}S_{a} \\
\left(\mathrm{~m}^{2}\right)\end{array}$ & $\begin{array}{c}S_{m} \\
\left(\mathrm{~m}^{2}\right)\end{array}$ & $\begin{array}{c}L \\
(\mathrm{~m})\end{array}$ & $\begin{array}{c}C \\
(\mathrm{~m})\end{array}$ \\
\hline$V I$ & 0 & $\infty$ & 0,0380 & 0,0045 & $\infty$ & 0,1125 \\
\hline$I$ & 0 & 6 & 0,0380 & 0,0044 & 0,3375 & 0,1125 \\
\hline$I V$ & 30 & 4,14 & 0,0349 & 0,0036 & 0,2690 & 0,1299 \\
\hline$I I$ & 0 & 4 & 0,0253 & 0,0030 & 0,2250 & 0,1125 \\
\hline$V$ & 30 & 2,64 & 0,0223 & 0,0023 & 0,1715 & 0,1299 \\
\hline$I I 1$ & 0 & 2 & 0,0126 & 0,0015 & 0,1125 & 0,1125 \\
\hline
\end{tabular}




\subsection{Mode opératoire}

La première configuration étudiée a été celle de l'aile bidimensionnelle en vue de comparer les résultats, d'une part, avec ceux qui avaient été obtenus avec le même profil sur le tunnel hydrodynamique de l'I.M.G., d'autre part, avec les résultats déduits du calcul [7].

Les autres configurations concernant les ailes tridimensionnelles ont été étudiées de la manière suivante : à vitesse constante de $7,5 \mathrm{~m} / \mathrm{sec}$, la pression de la surface libre a été réglée de façon qu'on obtienne différentes valeurs du nombre de cavitation; pour chaque nombre de cavitation, on a effectué un balayage en incidence en vue d'obtenir la courbe du coefficient de portance en fonction de l'incidence avec, aux extrémités, les limites correspondant au début de la cavitation sur l'extrados et sur l'intrados. Il faut noter que le début de la cavitation est accompagné d'un phénomène d'hystérésis déjà connu : dans ces essais, l'angle d'incidence correspondant au début de la cavitation est mesuré dans le sens croissant à l'extrados, dans le sens décroissant à l'intrados. Ces balayages en incidence à différentes valeurs du nombre de cavitation ont permis de définir pour chaque configuration d'aile le domaine de non cavitation dans le plan $\left(C_{L}, \sigma\right)$. Le domaine de non cavitation ainsi obtenu correspond à un nombre de ventilation $K_{o}$ donné (valeur du nombre de ventilation réglée à l'incidence $O$ ). Nous avons tenté de déterminer l'influence de ce nombre de ventilation $K$ sur la fourchette de non cavitation. C'est la raison pour laquelle nous avons choisi d'étudier la plupart des configurations à deux nombres de ventilation différents $(K=0,025$ et $K=0,050)$.

La naissance de la cavitation dépend d'un grand nombre de facteurs physiques parmi lesquels la teneur en air de l'eau joue un rôle prépondérant, mais avec le tunnel hydrodynamique que nous disposions, il n'était pas possible de régler la teneur en air indépendamment des autres paramètres et en particulier de la pression à la surface libre. Par conséquent, la teneur en air était fixée par les conditions de fonctionnement du tunnel et principalement par la valeur du nombre de cavitation. La durée de mise en route du tunnel est déterminante pour fixer la teneur en air ; cette demière décroît asymptotiquement en fonction du temps.

Nous avons relevé la teneur en air à l'aide d'un appareil Beckman pour chaque point de mesure et en jouant sur la valeur initiale de la teneur en air, on a tenté de mettre en évidence l'influence de cette demière sur le domaine de non cavitation.

La profondeur d'immersion dans tous les essais est de 1,1 corde, soit $123,7 \mathrm{~mm}$.

Dans le tableau II, on a rapporté les valeurs de la pression à la surface libre correspondant aux différentes valeurs du nombre de cavitation, pour les conditions de fonctionnement choisies.

\section{Tableau $\|$}

$h_{1}=123,7 \mathrm{~mm} \quad V=7,5 \mathrm{~m} / \mathrm{sec} \quad P_{V}\left(T=17^{\circ} \mathrm{C}\right)=1928,5 \mathrm{~Pa}$

\begin{tabular}{|c|r|r|r|r|r|r|r|r|}
\hline$\sigma$ & 0,09 & 0,1 & 0,12 & 0,15 & 0,2 & 0,3 & 0,4 & 0,5 \\
\hline $\begin{array}{c}P_{0} \\
(\mathrm{~mm} \mathrm{Hg})\end{array}$ & 24,4 & 26,5 & 30,7 & 37,1 & 47,7 & 68,7 & 89,9 & 111,0 \\
\hline
\end{tabular}

Avec les valeurs de la vitesse et de la profondeur d'immersion indiquées ci-dessous, on trouve comme différence de pression entre la surface libre et la cavité :

$$
P=P_{0}-P_{c}=-5,2\left[\mathrm{~cm} \mathrm{H}_{2} \mathrm{O}\right] \text { pour } K=0,025
$$

et

$$
P=P_{0}-P_{c}=+2,0\left[\mathrm{~cm} \mathrm{H}_{2} \mathrm{O}\right] \text { pour } K=0,050
$$

\section{Etude théorique}

La méthode de calcul mise en oeuvre dans cette étude est basée sur la technique des singularités qui permet de traiter l'écoulement tridimensionnel d'un fluide parfait et incompressible d'une aile dans le cadre des écoulements à potentiel. Le milieu est supposé semi infini, l'aile étant placée à une profondeur d'immersion suffisante pour que le traitement de la surface libre soit possible par la méthode des images.

Le calcul de l'aile supercavitante est rendu possible par la modélisation mathématique de la cavité. Les conditions aux limites sur la surface de l'aile se traduisent par une condition de glissement (condition du type Neumann). La frontière géométrique de la cavité devant être une surface de courant à pression constante, la condition sur celle-ci se traduit par une condition de vitesse tangentielle. En supposant dans un premier temps la géométrie de la cavité connue, on obtient la solution du problème en utilisant une répartition de simple couche sur l'aile (sources), une répartition de double couche sur la cavité (doublets tangentiels), une répartition de double couche sur l'aile, la cavité et le sillage (doublets normaux), plus une répartition de puits linéiques placée à l'arrière de la cavité dont l'intensité totale est égale à la somme des sources sur l'aile. Sur le sillage supposé indéformable et la cavité, la densité de doublets normaux est caractérisée par une loi suivant l'envergure de l'aile : cette loi couplée à une loi linéaire suivant la corde permet de définir la densité de doublets normaux sur toute la superficie de l'aile. Le calcul de la forme exacte de la cavité nécessite une procédure itérative qui permet d'obtenir celle-ci numériquement point par point. En raison de la présence des puits à l'arrière de la cavité, celle-ci ne se ferme pas complètement; de fait le modèle choisi est un modèle à cavité quasi fermée.



Figure 4 - Schématisation des frontières de l'écoulement 


\subsection{Formulation du problème}

L'aile est positionnée dans le repère (Oxyz) tel que la viteșse à l'infini amont $\vec{V}_{\infty}=\left|\vec{V}_{\infty}\right| \vec{j}$ soit parallèle à l'axe $\overrightarrow{O y}$ (fig. 4). L'aile, la cavité et le sillage sont des surfaces symétriques par rapport au plan (Oyz).

Les conditions aux limites se traduisent par les équations intégrales suivantes :

$$
\begin{aligned}
& \int_{\text {Scorps }} \sigma_{P} \vec{X}_{\sigma} \cdot \vec{n}_{M} d S_{P}+H_{\sigma}^{\circ} \sigma_{M}+\iint_{\mathrm{Scav}} \mu_{t P} \vec{X}_{t} \cdot \vec{n}_{M} d S_{P}+\int_{\text {Scorps, Scav }, \Sigma} \mu_{n P} \vec{X}_{n} \cdot \vec{n}_{M} d S_{P}+H_{D n}^{\circ} \mu_{n M} \\
& +\int_{L_{\text {Fcav }}} \lambda_{P} \vec{X}_{\sigma} \cdot \vec{n}_{M} d s_{P}=-\left|\vec{V}_{\infty}\right| \vec{j} \cdot \vec{n}_{M} \quad \text { pour } M \in S_{\text {corps }}
\end{aligned}
$$

$\iint_{\text {Scorps }} \sigma_{P} \vec{X}_{\sigma} \cdot \vec{t}_{M} d S_{P}+\underbrace{}_{\text {Scav }} \mu_{t P} \vec{X}_{t} \cdot \vec{t}_{M} d S_{P}+H_{D t}^{\circ} \mu_{t M}+\int_{\text {Scorps, Scav, } \Sigma} \mu_{n P} \vec{X}_{n} \cdot \vec{t}_{M} d S_{P}-\frac{1}{2}{\overrightarrow{\operatorname{grad}_{M}}}_{\mu_{n M}} \cdot \vec{t}_{M}$

$$
+H_{D n}^{1} \mu_{n_{M}}+\int_{L_{F c a v}} \lambda_{P} \overrightarrow{X_{\sigma}} \cdot \overrightarrow{t_{M}} d S_{P}=\left|\vec{V}_{\infty}\right|\left(\in_{M} \sqrt{1+K}-\vec{j} \cdot \overrightarrow{t_{M}}\right) \quad \text { pour } M \in S_{\text {cav }}
$$

Dans les expressions (1) et (2) les vecteurs $\vec{X}$ correspondent à l'influence "vitesse" des singularités correspondantes. Les paramètres $H$ représentent la contribution de la singularité correspondante sur elle-même.

$$
K=\frac{P_{\infty}-P_{\mathrm{cav}}}{\frac{1}{2} \rho \vec{V}_{\infty}^{2}}
$$

est le nombre de ventilation; $p_{\infty}$ étant la pression du fluide à l'infini amont à la profondeur d'immersion $h$. La condition de débit intérieur nul se traduit par la relation :

$$
\int_{\mathrm{L}_{\mathrm{Fcav}}} \lambda_{P} d S_{P}=\iint_{\mathrm{S}_{\mathrm{corps}}} \sigma_{P} d S_{P}
$$

La sortie tangentielle du fluide au bord de fuite de l'aile est assurée pour tous les points de cette courbe n'appartenant pas au saumon de l'aile :

$$
(\vec{v} \cdot \vec{n})_{M}=-\left|\vec{V}_{\infty}\right| \vec{j} \cdot \vec{n}_{M} \quad M \in L_{\mathrm{Fui}}
$$

La discrétisation du problème est réalisée par découpage de l'aile suivie de la cavité et du sillage en bandes parallèles au plan $(\mathrm{Oyz})$. On voit que le problème ainsi posé conduit à des nombres de ventilation $K$ variant suivant l'envergure de l'aile; l'uniformisation de ces valeurs permet d'obtenir la longueur de cavité en fonction de l'envergure de l'aile. Les équations (1), (2), (3), (4) discrétisées conduisent à un système linéaire de grande taille nécessitant une résolution par blocs; l'algorithme itératif utilisé sur les blocs est du type Gauss-Seidel, chaque bloc étant traité par la méthode de Householder. La formulation du problème suppose connue la forme géométrique de la cavité et la direction tangentielle du vecteur vitesse sur celle-ci. La cavité et le sillage sont initialisés par une surface cylindrique dont les génératrices sont parallèles à la vitesse à l'infini $\vec{V}_{\infty}$ et s'appuient sur le bord de fuite de l'aile. Sur cette forme initiale de cavité, la direction initiale de la vitesse tangentielle est prise parallèle à la vitesse $\vec{V}_{\infty}$ Les premières étapes permettent d'uniformiser les valeurs des nombres de ventilation par ajustement de la longueur de cavité suivant l'envergure de l'aile. En général 3 étapes de calcul sont suffisantes pour obtenir la forme en plan de la cavité. Repartant alors de cette configuration, on effectue une étape de calcul supplémentaire pour préciser davantage la forme de cavité.

\section{Résultats}

\subsection{Aile droite $A=6 ; \theta=0^{\circ}$.}

Cette configuration correspond à la maquette avec sa longueur maximale. On a cherché à déterminer la fourchette de non cavitation et linfluence du nombre de ventilation sur celle-ci. On a procédé de la manière suivante : à vitesse constante de $7,5 \mathrm{~m} / \mathrm{sec}$, la pression de la veine est réglée à chaque fois pour fixer la valeur du nombre de cavitation. Cette valeur peut varier entre une valeur minimale de l'ordre de 0,089 qui dépend des conditions de fonctionnement du tunnel, et une valeur maximale choisie égale à 0.5 .

Le domaine de non cavitation est défini dans le plan : $\left(C_{L}\right)$ limite, $\sigma$.

Pour évaluer l'influence du nombre de ventilation sur la fourchette de non cavitation, on a effectué les essais pour deux valeurs du nombre de ventilation $(K=0,025$ et $K=0,050$ à l'incidence nulle). La mise en incidence de l'aile se fait à débit d'air fixé. Dans ces conditions, le nombre de ventilation varie légèrement avec l'incidence. Les valeurs prises pour référence de chaque essai correspondent à $\alpha=0$ (voir paragraphe II.2).

\section{Configuration des cavités}

L'écoulement tridimensionnel autour d'une aile se caractérise principalement par la présence d'une cavité marginale à l'extrémité de l'aile. Cette cavité qui résulte de la composante transverse de l'écoulement se distingue de plus en plus de la cavité principale lorsque l'incidence du profil augmente. L'évolution de la configuration des cavités en incidence est dominée par ce fait. Il existe toujours une cavité principale, prépondérante à l'incidence nulle et lorsque l'incidence augmente, l'accroissement de la variation de circulation le long de l'envergure modifie la configuration de l'écoulement de façon à augmenter l'effet de la tridimensionnalité. 
A titre d'exemple, on décrit l'évolution de la configuration des cavités en incidence dans le cas de la figure $5(\sigma=0,5$ et $K=0,025)$.

A l'incidence nulle, la cavité principale a une longueur de $95 \mathrm{~cm}$, avec une légère excroissance vers l'extrémité qui va donner naissance à la cavité marginale. Une cavité marginale apparaît dès que le profil est mis en incidence. A une incidence de $0,6^{\circ}$ la longueur de cette cavité marginale est de $75 \mathrm{~cm}$. La cavité principale en même temps s'est raccourcie à une longueur de $80 \mathrm{~cm}$. Lorsqu'on augmente l'incidence, la cavité marginale a tendance à devenir prépondérante. Elle absorbe la plus grande partie de l'air injecté et se prolonge de plus en plus tandis que la cavité principale se raccourcit considérablement. A une incidence de $3,6^{\circ}$ la longueur de la cavité marginale est de $80 \mathrm{~cm}$ et celle de la cavité principale de $35 \mathrm{~cm}$. Dans cet exemple, l'incidence correspondant au début de cavitation sur l'extrados est de $3,6^{\circ}$.

L'évolution des longueurs des deux cavités dépend fortement du nombre de ventilation $\left(K_{0}\right)$. Lorsqu'on l'augmente, on diminue la longueur de la cavité à l'incidence nulle; l'évolution de la cavité marginale, lors de la mise en incidence du profil, est alors bien plus importante et par contre celle de la cavité principale est moins étendue. A titre d'exemple, pour le balayage en incidence correspondant à la figure $6(\sigma=0,5$ et $K=$ 0,050 ), la cavité principale se raccourcit de $36 \mathrm{~cm}$ (à l'incidence zéro), à $29 \mathrm{~cm}$ (à l'incidence $3,6^{\circ}$ ) et en même temps, la cavité marginale s'allonge de $27 \mathrm{~cm}$ (à l'incidence $1^{\circ}$ ), à $54 \mathrm{~cm}$ (à l'incidence de $3,6^{\circ}$ ).

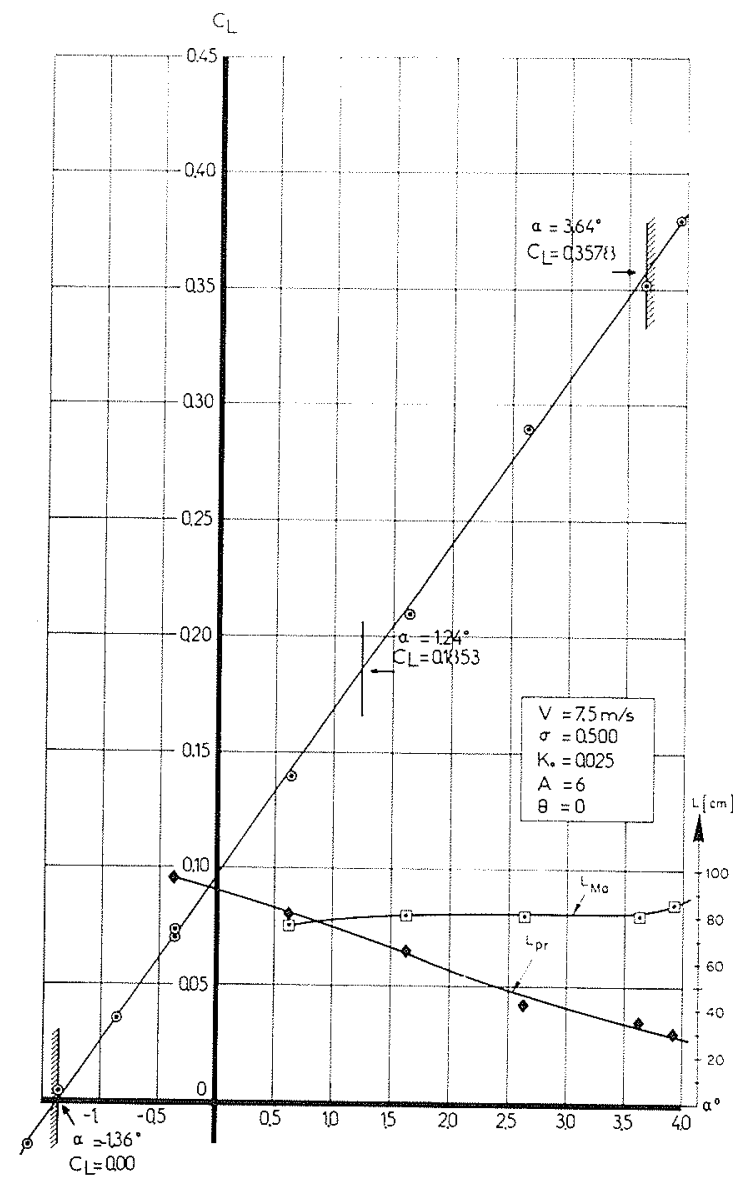

Figure 5 - Cofficient de portance en fonction de l'incidence.

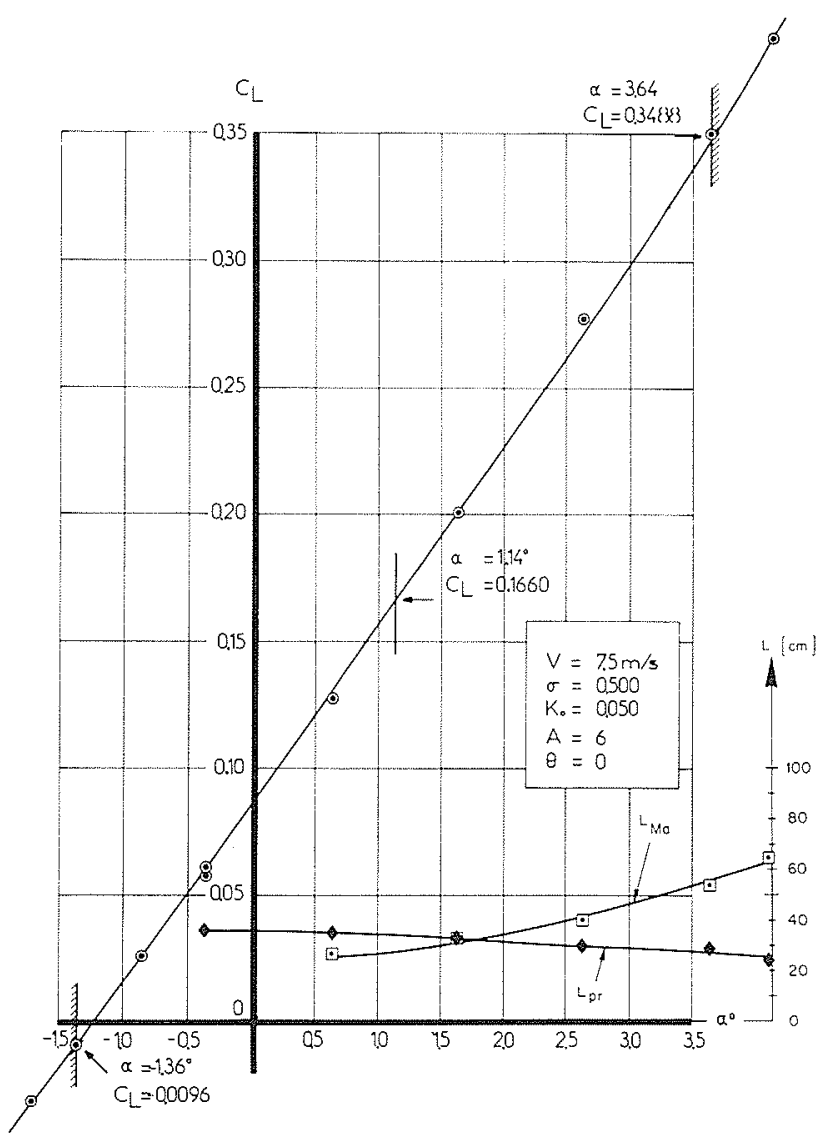

Figure 6 - Coefficient de portance en fonction de l'incidence.

La cavité marginale se ferme souvent par un jet rentrant mais lorsqu'elle se prolonge loin en aval, le mouvement tourbillonnaire dont elle est dotée atténue l'importance de ce jet. Elle est presque toujours suivie d'un tourbillon cavitant qui se propage très loin derrière l'aile.

Lorsque l'aile se trouve placée à une incidence négative inférieure en valeur absolue à la limite de cavitation sur l'intrados, la configuration de l'écoulement ne varie pas considérablement par rapport à celle qui correspond à l'incidence nulle. La cavité marginale ne se forme pas, même jusqu'au dénoyement de l'intrados. Il faut signaler cependant que l'incidence de l'aile correspondant au début de la cavitation sur l'intrados est beaucoup plus proche de celle qui correspond au dénoyement total du même intrados, que l'incidence correspondant au début de cavitation à l'extrados ne l'est du dénoyement à l'extrados. Cela ne favorise pas la formation d'une cavité marginale à l'extrémité de l'aile. Toutefois, à l'incidence négative il existe néanmoins des filets tourbillonnaires à l'extrémité de l'aile qui se prolongent loin à l'aval mais ne forment pas une véritable cavité.

\section{Influence du nombre de cavitation $\sigma$}

Le comportement de l'aile au début de la cavitation est bien différent à l'intrados et à l'extrados. Le nómbre de cavitation $\sigma$ joue un rôle déterminant.

Lorsque le nombre de cavitation est assez grand $(0,4-0,5)$ à l'extrados, la cavitation apparaît sous forme d'une lame assez mince près du bord d'attaque, qui s'étend en profondeur et suivant l'envergure au fur 
et à mesure que l'incidence augmente. La zone où cette cavitation apparaît se situe environ aux deux tiers de l'envergure. A l'intrados la cavitation apparaît de la même façon, mais à une incidence plus petite en valeur absolue (de l'ordre de quelques dixièmes de degré). Elle commence en général à apparaître près de l'encastrement de l'aile et lorsque l'incidence diminue, elle s'étend vers l'extrémité. Au fur et à mesure du développement, la cavitation reste toujours plus étendue en profondeur au voisinage de l'encastrement qu'à l'extrémité.

Lorsque le nombre de cavitation est assez petit $(0,09-0,2)$ l'apparition de la cavitation sur l'extrados est très brutale sous forme d'une cavité bien développée et prolongée vers l'aval qui envahit tout l'extrados lorsque l'incidence croît. Cette cavité s'accroche au voisinage du bord d'attaque et le moindre défaut sur l'aile dans cette zone peut faciliter la formation de celle-ci. Il en est de même lorsque des particules en suspension dans l'eau se collent à proximité du bord d'attaque, peut-être sous l'influence du gradient de pression assez considérable qui existe à cet endroit.

L'influence du nombre de cavitation sur la cavitation à l'intrados est par contre beaucoup moins importante. En dehors du fait qu'en diminuant le nombre de cavitation on augmente l'indétermination de l'apparition de la cavitation sur l'intrados, on ne change ni la forme ni l'endroit de l'appararition de cette cavitation.

Lorsqu'on augmente l'incidence, on augmente la perturabation de l'écoulement dans la zone de l'encastrement de la maquette. Dans cette zone, les couches limites engendrées par la paroi et par l'aile se combinent et forment un écoulement fortement perturbé. La perturbation est d'autant plus accentuée que l'incidence du profil est plus grande. Cela favorise la formation de petites bulles de vapeur, surtout sur l'extrados, qui sont ensuite happées par l'écoulement. Ce phénomène est concomitant à une légère diminution de la portance lorsque le nombre de cavitation est assez petit et l'incidence suffisamment grande (fig. 7).

Pente de la courbe $C_{L}(\alpha)$ : coefficient $C_{L} \alpha$

Le coefficient $C_{L \alpha}$, obtenu d'après la formule :

$$
C_{L \alpha}=a_{0} A /\left(A+1+a_{0} / \pi\right)
$$

est égal à 3,67 $\mathrm{rad}^{-1}$ avec $a_{0}$ : pente (en bidimensionnel) égale à $5,33 \operatorname{rad}^{-1}$ et $A$ : allongement du profil égal à 6 .

Sur les courbes expérimentales on obtient une valeur moyenne de l'ordre de $3,72 \mathrm{rad}^{-1}$ pour ce coefficient, ce qui confirme le résultat de la formule précédente à $1,3 \%$ près.

\subsection{Aile droite $A=4 ; \theta=0^{\circ}$}

Cette configuration correspond pour la maquette à une longueur de 2 cordes. Le nombre de cavitation varie de 0,088 à 0,5 . Le nombre de ventilation comme dans le cas précédent vaut soit 0,025 soit 0,050 . En plus, nous avons étudié le coefficient de portance en fonction du nombre de ventilation. On s'est intéressé aussi à la teneur en air de l'eau dans les conditions de fonctionnement propres à chaque essai. La teneur en air de l'eau expri-

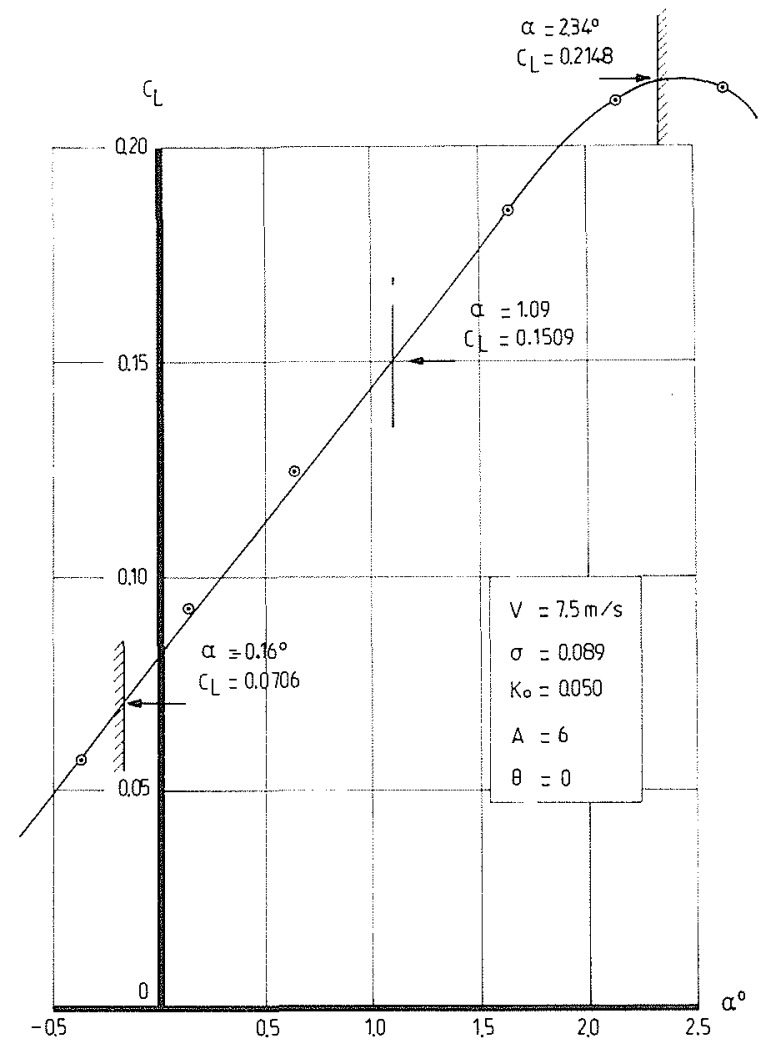

Figure 7 - Coefficient de portance en fonction de l'incidence.

mée en pourcentage par rapport au pourcentage de la saturation a été mesurée spécialement au début de la cavitation sur l'extrados et sur l'intrados. On a essayé de dégager l'influence de la teneur en air indépendamment des autres paramètres en jouant sur la durée de mise en route de l'installation qui est un paramètre assez important pour la détermination de la teneur en air de l'eau.

Configuration des cavités

D’une façon générale, la configuration des cavités et les phénomènes physiques qui se produisent lorsqu'on modifie le nombre de cavitation et l'incidence de l'aile sont plus ou moins identiques à ceux de la configuration précédente $(A=6-\theta=0)$.

On a remarqué qu'à faible incidence la cavité marginale se développe à partir de l'extrémité de l'aile et du bord de fuite. Lorsque l'incidence de l'aile continue à augmenter, la cavité marginale remonte de plus en plus sur l'extrados. Ce mécanisme de remontée de la cavité marginale sur l'extrados est beaucoup plus net dans le cas présent que dans le cas précédent correspondant à un plus grand allongement.

Le nombre de cavitation est aussi un paramètre influant sur ce phénomène : lorsque le nombre de cavitation est assez grand, la cavité remonte sur l'extrados avec une incidence plus grande que lorsque le nombre de cavitation est petit. Suivant les valeurs respectives du nombre de ventilation et du nombre de cavitation, ce phénomène peut entraîner une diminution assez considérable de la portance.

La figure 18 a montre la forme en plan de la cavité obtenue par le calcul et l'expérience. Nous constatons 


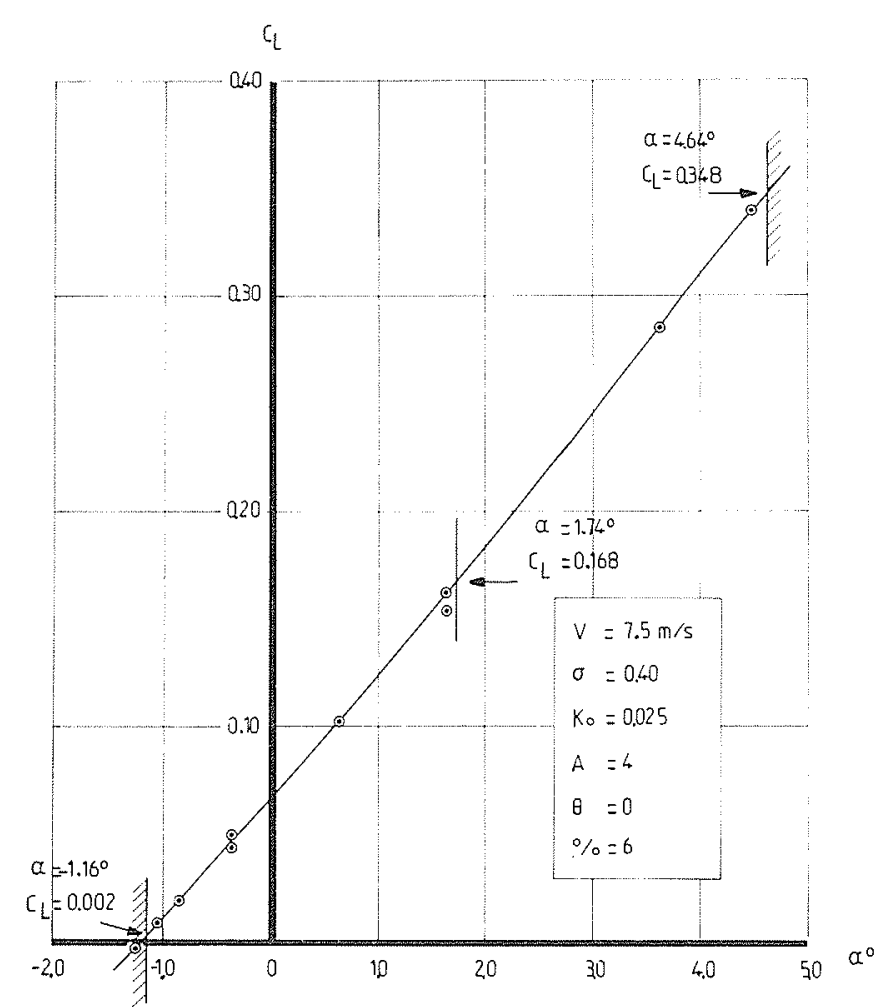

Figure 8 - Coefficient de portance en fonction de l'incidence.

que la forme expérimentale posséde une partie rentrante beaucoup plus accentuée que la forme théorique. La cavité expérimentale est plus étendue que la cavité théorique ; d'autre part, nous remarquons que le calcul ne donne qu'une approximation de la cavité marginale. La figure $18 \mathrm{~b}$ montre une vue tridimensionnelle du résultat théorique; le gonflement de la cavité marginale est conforme aux observations faites en tunnel hydrodynamique.

\section{Influence du nombre de cavitation}

Les résultats des balayages en incidence sont assez semblables à ceux de la configuration précédente $(A=$ $6, \theta=0$ ). Les courbes sont pratiquement des droites lorsque le nombre de cavitation est assez grand $(0,4-$ $0,5)$ et elles sont convexes lorsque le nombre de cavitation est compris entre 0,2 et 0,09 (figs. 8 et 9 ).

Lorsque l'envergure d'une aile est diminuée, on doit s'attendre à ce que l'écoulement transverse et la variation de la circulation le long de l'envergure deviennent plus importants. Il s'ensuit une diminution de la portance.

Une comparaison des résultats montre qu'effectivement dans les mêmes conditions de fonctionnement que précédemment $(A=6)$, le coefficient de portance est diminué.

La figure 10 montre l'évolution du coefficient de portance en fonction du nombre de ventilation. Lorsque le nombre de ventilation croît, le coefficient de portance diminue jusqu'à une valeur du nombre de ventilation proche de 0,07 , ce qui correspond à une longueur de cavité de l'ordre de $23 \mathrm{~cm}$. A partir de ce point, lorsqu'on continue à augmenter la valeur du nombre de ventilation (la longueur de la cavité diminue), le coeffi-

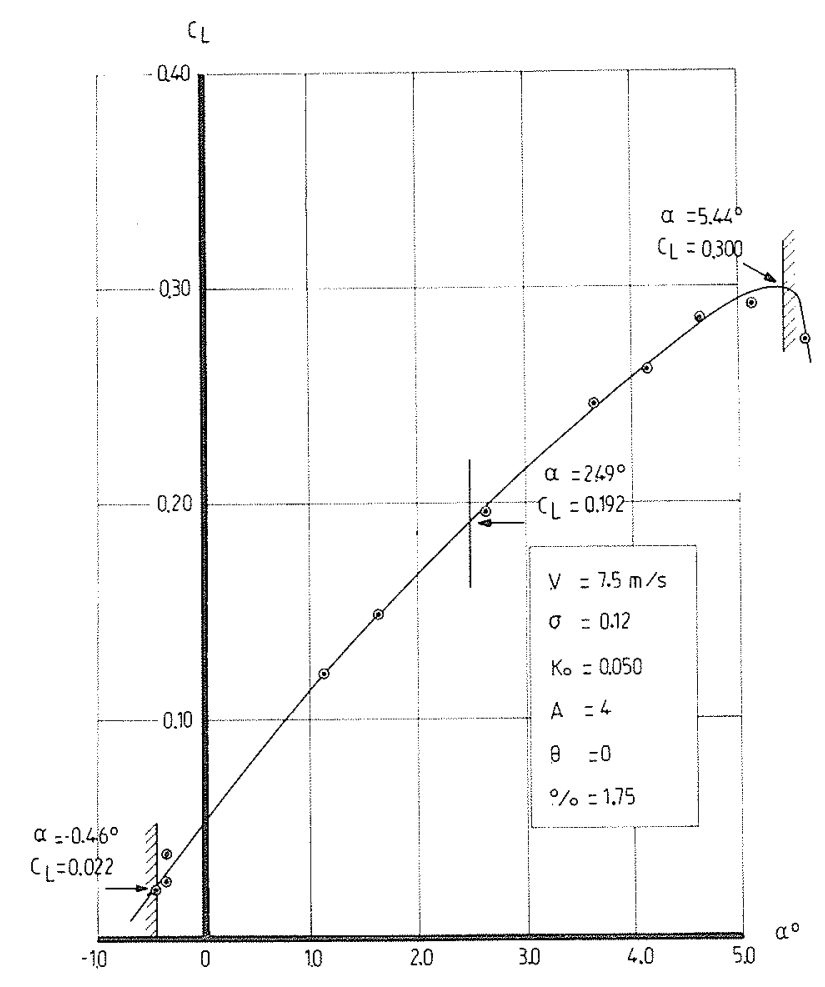

Figure 9 - Coefficient de portance en fonction de l'incidence.

cient de portance croît. On retrouve donc le minimum déjà observé sur les écoulements bidimensionnels, dans les études antérieures. On remarque que dans ce cas l'extrados est complètement mouillé.

Pente de la courbe $C_{L}^{\prime \prime \prime}(\alpha)$ : coefficient $C_{L \alpha}$

Le coefficient $C_{L \alpha}$ obtenu d'après la formule :

$C_{L \alpha}=a_{o} A /\left(A+1+a_{o} / \pi\right) \quad$ est égal à 3,18 $\mathrm{rad}^{-1}$

D'après les courbes expérimentales, on obtient une valeur moyenne de l'ordre de $3,23 \mathrm{rad}^{-1}$, ce qui confirme le résultat de la formule précédente à $1,3 \%$ près.

\subsection{Aile droite $A=2, \theta=0^{\circ}$}

Cette configuration correspond pour la maquette à une longueur de 1 corde. Le nombre de cavitation varie de 0,092 à 0,5 , le nombre de ventilation vaut 0,050 dans tous les essais. On a également examiné l'évolu. tion du coefficient de portance en fonction du nombre de ventilation.

\section{Commentaires sur les résultats}

La diminution de l'allongement entraine une diminution assez considérable du coefficient de portance et une augmentation importante de l'angle limite de cavitation sur l'extrados; on passe ainsi d'un angle limite de l'ordre de 3-4 degrés à l'allongement $A=6$ à un angle limite de l'ordre de 7-8 degrés à $A=2$.

L'augmentation de cet angle limite permet de mieux examiner les phénomènes marginaux qui se produisent à forte incidence aux petits nombres de cavitation. D'une manière générale, on peut insister sur le fait que la diminution de l'allongement a pour effet d'augmenter 


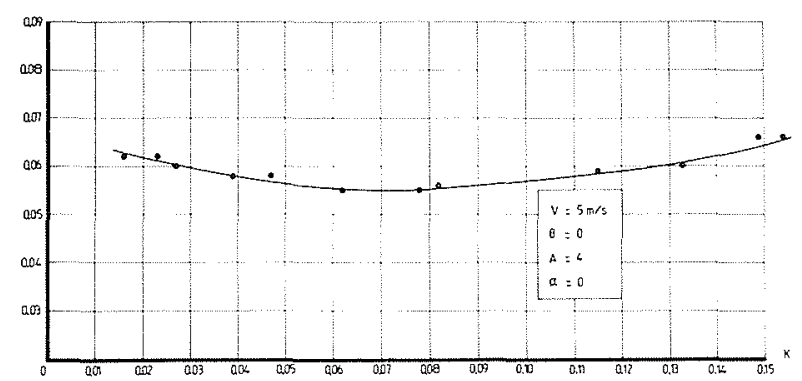

Figure $10-$ Coefficient de portance en fonction du nombre de ventilation $-A=4$.

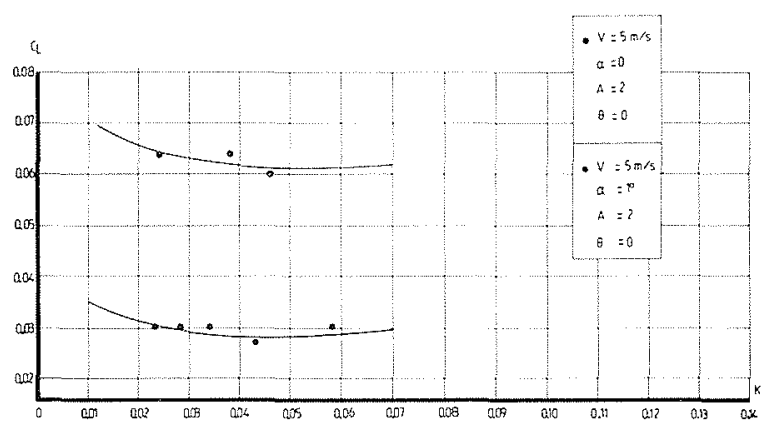

Figure 11 - Coefficient de portance en fonction du nombre de ventilation $-A=2$.

la tridimensionnalité de l'écoulement et les phénomènes associés.

A propos de la configuration des cavités, on constate que pour le nombre de cavitation $\sigma=0,5$ la cavité marginale ne remonte sur l'extrados que pour une incidence supérieure à $7^{\circ}$, après apparition de la cavitation sur le bord d'attaque. Sur ce phénomène il existe une hystérésis qui n'est pas négligeable; à titre d'exemple lorsque dans le cas précédent on diminue l'incidence, la cavité marginale reste sur l'extrados jusqu'à une incidence de $3,2^{\circ}$ environ.

Aux petits nombres de cavitation $(\sigma=0,09-0,2)$ l'effet d'encastrement est important. A nombre de cavitation $\sigma=0,09$, cette cavitation forme presque une cavité à l'encastrement du profil.

Le coefficient $C_{L \alpha}$ d'après la formule mentionnée précédemment vaut $C_{L \alpha}=2,27 \mathrm{rad}^{-1}$. D'après les courbes expérimentales, on obtient une valeur moyenne de l'ordre de 2,29 $\mathrm{rad}^{-1}$ pour ce cœefficient.

La figure 11 est relative à l'évolution du coefficient de portance en fonction du nombre de ventilation. L'allure générale de la courbe est identique au cas précédent, mais les valeurs de la portance sont de l'ordre de la moitié de celles qui correspondent à l'allongement 4.

\subsection{Aile en flèche $A=4 ; 14 ; \theta=30^{\circ}$}

Cette configuration correspond pour la maquette à une longueur de $269 \mathrm{~mm}$ mesurée perpendiculairement à l'axe de la veine (fig. 3). Le nombre de cavitation varie de 0,086 à 0,5 . Le nombre de ventilation est égal à 0,050 dans tous les essais. On a étudié aussi l'évolution du coefficient de portance en fonction du nombre de ventilation pour deux vitesses de 5 et $7,5 \mathrm{~m} / \mathrm{s}$.

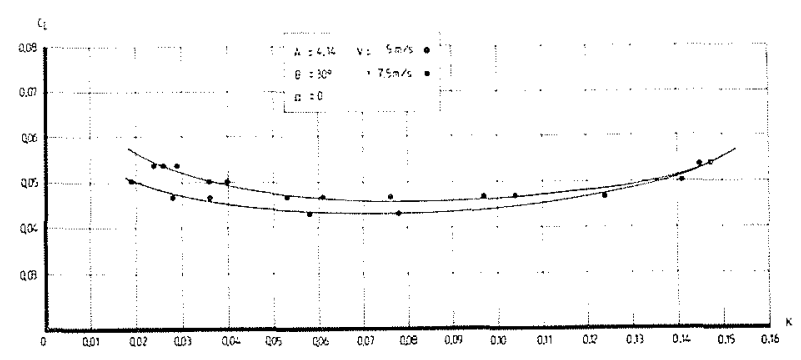

Figure 12 - Coefficient de portance en fonction du nombre de ventilation $-A=4.14$.

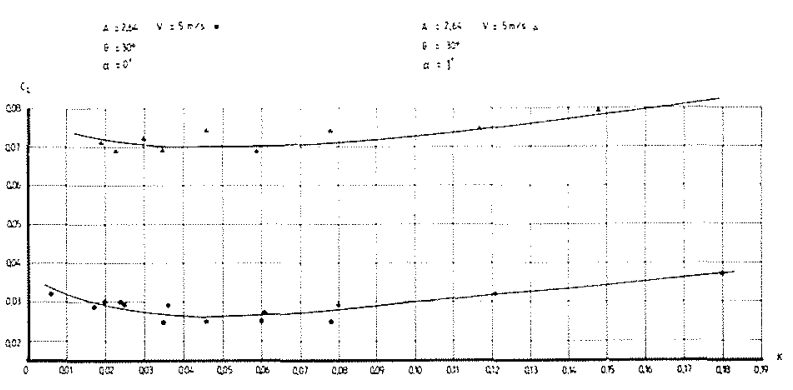

Figure 13 -Coefficient de portance en fonction du nombre de ventilation $-A=2.64$.

\section{Commentaires sur les résultats}

L'apparition de la cavitation sur l'extrados ou sur l'intrados s'effectue de la même façon que pour les ailes droites en fonction du nombre de cavitation. On constate qu'aux petits nombres de cavitation, lorsque l'aile est placée à une incidence proche de l'angle limite de cavitation à l'extrados, il apparaît par moment une cavité conique assez bien développée qui parcourt rapidement l'extrados vers l'extrémité de l'aile. Comme dans le cas de l'aile droite, cette cavité prend naissance en un point de l'extrados proche du bord d'attaque ; cependant, dans le cas présent, elle est beaucoup moins stable. A une incidence légèrement plus grande, cette cavité reste accrochée sur une largeur de 3 à $4 \mathrm{~cm}$ de l'extrados. En se référant au cas de l'aile droite on observe ainsi une autre différence : dans le cas de l'aile en flèche, lorsque la cavité devient stable, son origine n'est pas ponctuelle, mais elle est étendue sur une cer-

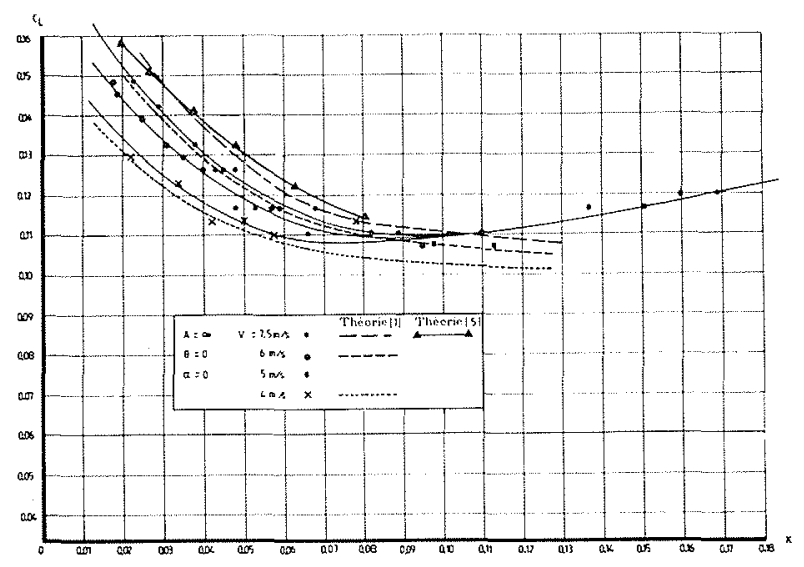

Figure 14 - Coefficient de portance en fonction du nombre de ventilation - Profil bidimensionnel. 
taine largeur de l'extrados. Les particules parasites de l'eau ne restent pas accrochées sur le bord d'attaque, elles sont entraînées vers l'extrémité de l'aile avec l'écoulement transverse.

On remarque aussi que la perturbation produite à l'encastrement du modèle, aux petits nombres de cavitation et aux fortes incidences, est sensiblement moins importante que pour les ailes droites et surtout que l'origine de ces perturbations se situe davantage vers la base du profil. La cavité marginale remonte sur l'extrados pour une incidence plus grande que dans le cas des ailes droites.

La figure 12 représente l'évolution du coefficient de portance en fonction du nombre de ventilation pour deux vitesses de 5 et $7,5 \mathrm{~m} / \mathrm{s}$. En comparant cette courbe avec celle qui correspond à l'aile droite $(A=4$, $\theta=0$ ), on constate une diminution du coefficient de portance pour l'aile en flèche. Le coefficient $C_{L \alpha}$ minimal diminue de 0,055 pour $\theta=0^{\circ}$ à 0,043 pour $\theta=30^{\circ}$.

\subsection{Aile en flèche $A=2,64 ; \theta=30^{\circ}$}

Cette configuration correspond pour la maquette à une longueur de $171,5 \mathrm{~mm}$ mesurée perpendiculairement à l'axe de la veine. La corde du profil vaut $129,9 \mathrm{~mm}$. Le nombre de cavitation varie de 0,082 à 0,5 . Le nombre de ventilation est égal à 0,050 dans tous les essais. L'évolution du coefficient de portance en fonction du nombre de ventilation a été aussi étudiée.

\section{Commentaires sur les résultats}

En ce qui concerne la configuration et la longueur des cavités ainsi que les phénomènes marginaux, l'aile en flèche ne diffère pas beaucoup par rapport à l'aile droite. Mais quantitativement la mise en flèche modifie notablement les résultats expérimentaux.

La courbe 13 est relative à l'évolution du coefficient de portance en fonction du nombre de ventilation aux deux incidences de $0^{\circ}$ et $1^{\circ}$. La dispersion des points

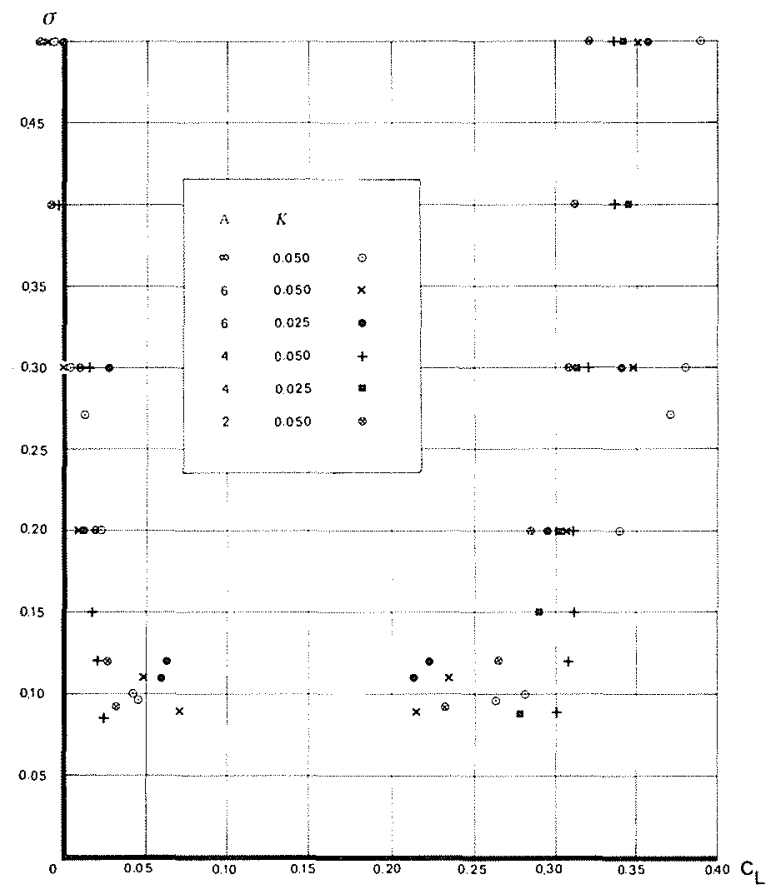

Figure 15 - Limites du domaine de non cavitation - Aile droite. de mesure est assez importante. Les faibles valeurs du coefficient de portance peuvent en être la cause. C'est la raison pour laquelle deux essais ont été effectués l'un avec l'incidence $0^{\circ}$ et l'autre avec une incidence de $1^{\circ}$.

\subsection{Validation des résultats}

Les essais effectués en écoulement bidimensionnel ont essentiellement eu pour but de valider les résultats par comparaison avec les théories [5] et [7]. La figure 14 montre une très bonne coincidence entre les deux théories dans le cas où le nombre de Froude est grand. $\mathrm{La}$ concordance avec l'expérience apparaît bonne, compte tenu d'une part de ce que les théories ne sont pas parfaites, d'autre part que l'incertitude affectant les points expérimentaux n'est pas négligeable. On remarque que pour les grandes valeurs du nombre de ventilation, la portance trouvée expérimentalement augmente assez rapidement, alors que la portance calculée devient inférieure.

\section{Doma ine de non cavitation}

L'un des buts de la présente étude est de déterminer l'influence de la forme en plan sur le domaine de non cavitation. Les calculs ont été effectués sur un ordinateur CYBER 750.

Sur le plan numérique, la difficulté essentielle réside dans le fait que nous ne pouvons pas fixer a priori le nombre de ventilation, celui-ci étant une inconnue du problème. En conséquence, nous avons fixé la longueur médiane de la cavité de façon à obtenir le nombre de ventilation. expérimental. Nous constatons que le domaine de non cavitation dans le plan $\left(C_{L}, \sigma\right)$ est pratiquement insensible à la présence de la surface libre: ce résultat est montré sur les courbes 16 et 17 . Cette remarque permet de traiter le problème numérique en milieu infini, ce qui rend l'exploitation beaucoup moins onéreuse.

Sur le plan expérimental, une étude précédente faite par A. Rowe [4] montrait que l'influence de la forme en plan peut être importante. Cependant, compte tenu de l'étroitesse de la veine où les essais auraient été effectués, une incertitude subsistait sur la validité des résultats [4]. Dans le cas présent, les dimensions de la veine du tunnel NEYRTEC garantissent que les effets de confinement ne sont pas déterminants; malheureusement ce tunnel ne permet pas de fixer la valeur de la teneur en air pour tous les essais effectués. Il s'ensuit une certaine dispersion des mesures, et là aussi, il faut être prudent sur l'interprétation des résultats.

Quelques tendances paraissent acquises (figs 15, 16, 17) :

1) Le domaine de non cavitation obtenu par le présent calcul est dans tous les cas plus étroit que celui obtenu expérimentalement ; il est possible de donner une intervrétation à cette différence :

- ia naissance de la cavitation dépend d'un grand nombre de facteurs physiques que le calcul ne prend pas en compte : la teneur en air, le nombre de germes et leur répartition en taille. 




Figure 16 - Limites du domaine de non cavitation - Aile en flèche $-=30^{\circ}$.

- l'influence de la couche limite n'est pas prise en compte ; le point d'apparition de la cavitation a été déterminé numériquement comme étant le point de coefficient de pression minimal.

2) Lorsque l'allongement de l'aile diminue, la fourchette de non cavitation diminue ; les figures 16 et 17 montrent que cette diminution s'accompagne d'un déplacement du centre de la fourchette vers les $C_{L}$ croissant pour le calcul, vers les $C_{L}$ décroissant pour l'expérience.

D’une manière générale, lorsque la flèche augmente, la fourchette de non cavitation diminue ; la comparaison des figures 16 et 17 montre que cette tendance est beaucoup plus marquée aux grandes valeurs du nombre de cavitation qu'aux petites. D'autre part, ces figures nous montrent que dans le présent calcul, l'effet de flèche s'accompagne d'un déplacement du domaine de non cavitation vers les $C_{L}$ décroissant.

3) Au nombre de cavitation $\sigma=0,1$, la fourchette de non cavitation obtenue par l'expérience est beaucoup plus grande que celle obtenue par le calcul. L'étendue de cette fourchette est certainement liée à la valeur de la teneur en air obtenue lors des essais, qui était toujours à peu près du même ordre de grandeur.

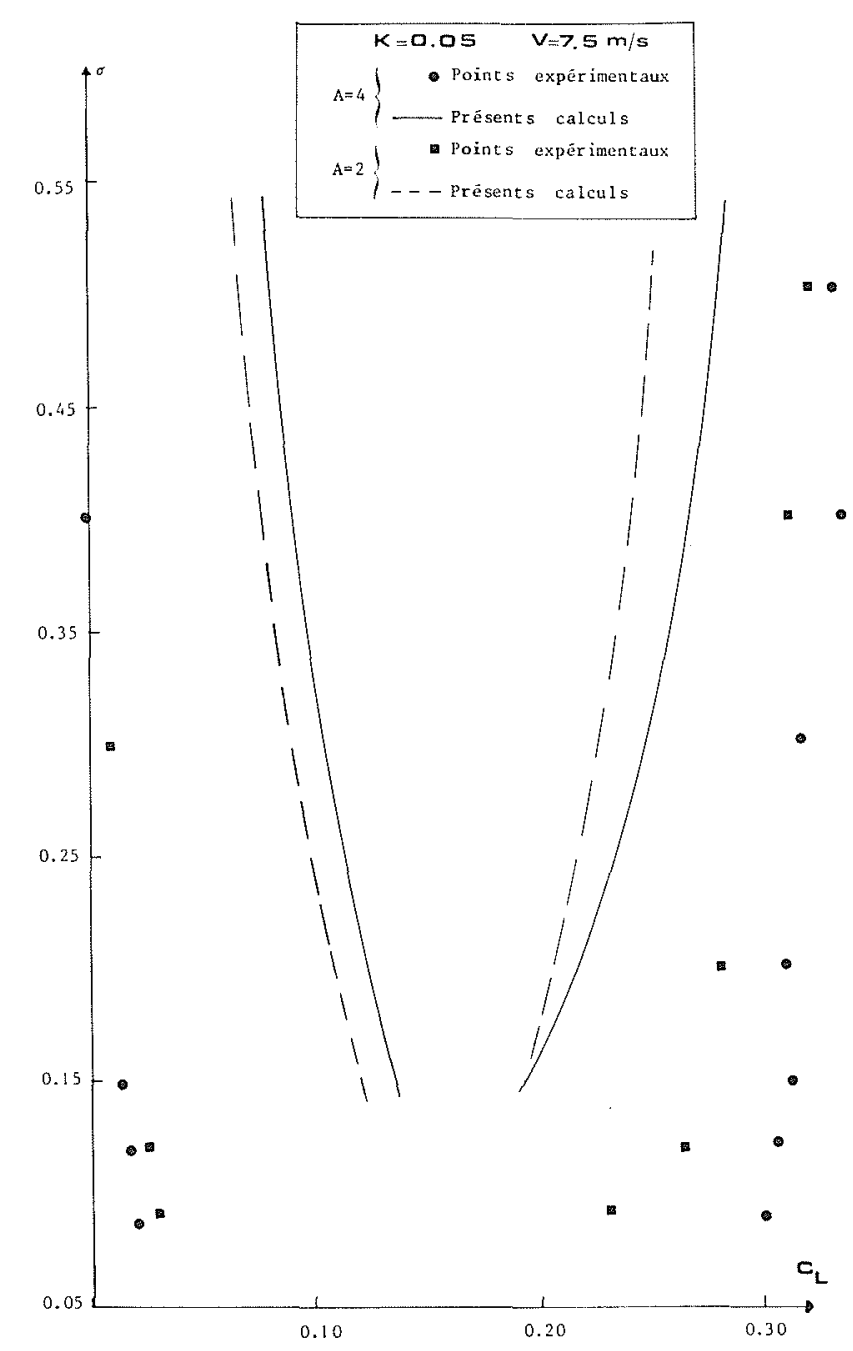

Figure 17 - Limites du domaine de non cavitation - Aile droite $-=0^{\circ}$.

On constate que la limite inférieure est proche de la portance nulle, ce qui est cohérent avec le fait qu'à l'adaptation, la portance de l'aile est positive. L'influence du nombre de ventilation paraît mineure et on ne constate pas une grande différence lorsque le nombre de ventilation passe de 0,025 à 0,05 ; pourtant le rapport entre les longueurs de la cavité est de l'ordre de 2,5. Cela est dû au fait que le soufflage a pour effet de modifier en même temps la portance et l'incidence d'adaptation.

\section{Bilan de l'étude}

Au terme de cette étude, il est possible de retenir quelques faits importants.

1) Lorsqu'on diminue l'allongement d'une aile à base ventilée, on diminue le coefficient de portance et le coefficient $C_{L \alpha}$. C'est un résultat classique pour les ailes subcavitantes, et il n'y aurait aucune raison pour qu'il ne reste pas vrai avec une aile à base ventilée. La mise en flèche de l'aile a également pour effet de diminuer le coefficient de portance.

2) Lorsque l'aile porte peu, la cavité marginale 


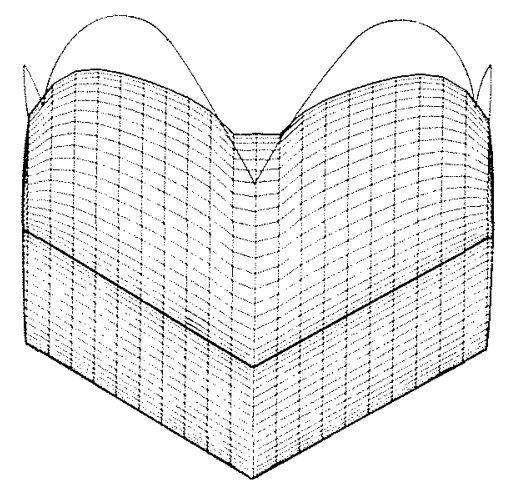

$$
\begin{aligned}
& \text { A I L E EN F L E H E } \\
& \Theta=30^{\circ}, \quad A=4,14 \\
& \text { Incidence } \alpha=0^{\circ}, 36 \\
& \text { Profondeur } d^{\prime} \text { immersion : 1corde }
\end{aligned}
$$

Figure $18 \mathrm{a}$ - Aile en flèche - Forme en plan $-\theta=30^{\circ}$; $A=14$ Incidence $\alpha=0^{\circ} 36$; Profondeur d'immersion : 1 corde.

s'accroche à la bosse de l'aile, puis lorsque l'incidence augmente, son extrémité remonte vers le bord d'attaque et rencontre peu à peu l'extrados. Ce comportement a été décrit précédemment en [4] où il a fait l'objet d'un dessin assez explicite. Il est d'autant plus net que l'allongement de l'aile est réduit.

3) En général la cavité expérimentale est plus étendue que la cavité théorique. D'autre part, par la nature même du calcul, la forme en plan de la cavité marginale est simplifiée par rapport à la réalisté. Le gonflement de la cavité
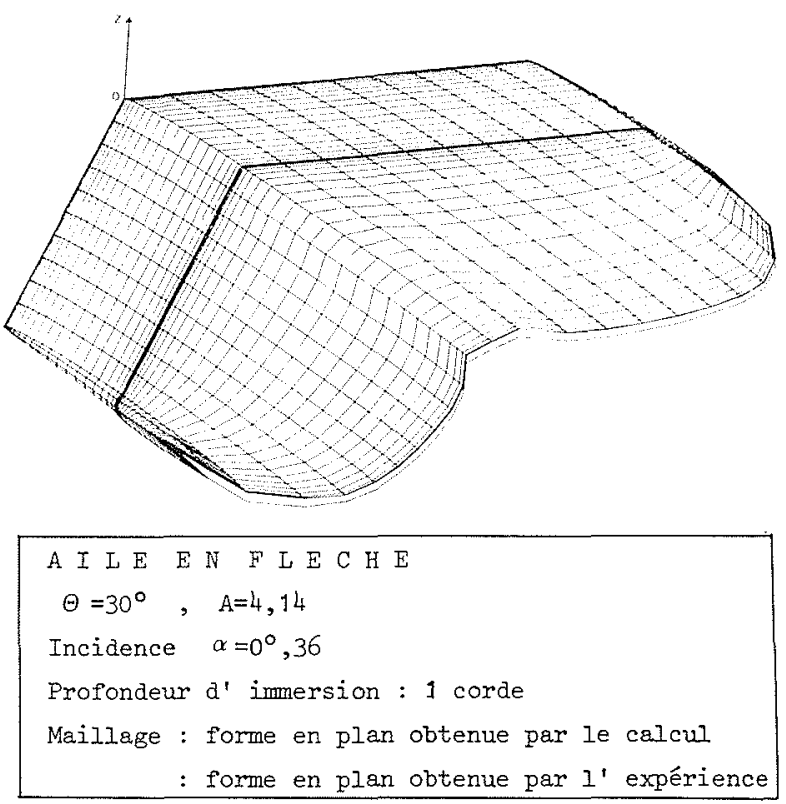

Figure $18 \mathrm{~b}$ - Aile en flèche - Vue tridimensionnelle $-\theta=30^{\circ}$; $A=4,14$ Incidence $\alpha=0^{\circ} 36$; Profondeur d'immersion : 1 corde Maillage $=$ forme en plan obtenue par le calcul et par l'expérience

marginale est conforme aux observations faites en tunnel hydrodynamique.

4) A l'extrados, la cavitation apparaît environ aux deux tiers de l'envergure pour gagner le reste de l'aile. A l'intrados, la cavitation apparaît d'abord à l'encastrement de l'aile. Ce résultat montre l'intérêt qu'il y aurait à utiliser une aile vrillée, le calage des sections de l'aile à l'extrémité étant plus faible qu'à l'encastrement.

5) La fourchette de non cavitation théorique est toujours plus étroite que la fourchette expérimentale.

\section{Références}

[1] MICHEL J.M. et ROWE A. - Profils minces supercavitants à arrière tronqué. Définition et étude théorique de profils portants, à nombre de ventilation nul, en présence d'une surface libre. La Houille Blanche, $\mathrm{n}^{\circ} 3,1974$.

[2] ROWE A.. et MICHEL J.M. - Two-dimensional base-vented hydrofoils near a free surface : influence of the ventilation number. Journal of Fluids Engineering, Special Section on cavity flows, December 1975, pp. 465-474.

[3] MICHEL J.M. - Wakes of developed cavities. Journal of Ship Research, vol. $21, \mathrm{n}^{\circ} 4,1977$, pp. 225-238.

[4] ROWE A. - Evaluation study of a three-speed hydrofoil with wetted upper-sides. Journal of Ship Research, vol. 23, $n^{\circ} 1,1979$, pp. 55-65.

[5] PELLONE C. - Application de la méthode des singularités au calcul des structures supercavitantes en théorie non linéaire. Thèse de Docteur-Ingénieur, I.M.G. Grenoble, 1981.

[6] PELLONE C. et ROWE A. - Etude des caractéristiques d'une aile à base ventilée en théorie non linéaire. $17^{\mathrm{e}}$ Colloque d'Aérodynamique Appliquée, Grenoble, 1980.

[7] ROWE A. and KUENY J.L - Recent research results on cavity flows about hydrofoils. $13^{\mathrm{e}}$ Symposium d'Hydrodynamique Navale, O.N.V.-S.R.A.J., Tokyo, Octobre 1980.

[8] PELLONE C. and ROWE A. - Supercavitating hydrofoils in non linear theory. Third International Conference on Numerical Ship Hydrodynamics, Paris, 16-19 Juin 1981.

[9] ROWE A. - Profils hydrodynamiques bidimensionnels à base ventilée en présence d'une surface libre. Ths̀e de Docteur-Ingénieur, I.M.G. Grenoble, 1973. 


\section{Discussion}

Président : J. DODU

M. le Président. - Merci de nous avoir présenté très rapidement beaucoup de choses. Je signale à l'assistance que j'a connu M. LAALI venant de l'Iran, son pays natal, et ne maîtrisant que très peu la langue française. Vous avez pu apprécier par la clarté de son exposé, les progrès accomplis.

M. LAALI. - précise que c'est M. PELLONE qui a mis au point cette méthode de calcul, et c'est lui qui répondra aux questions sur la partie numérique.

M. CORNIGLION. - Vous calculez l'écoulement autour de profil portant. Je n'ai pas bien saisi dans votre exposé comment vous avez généré la portance puisque vous utilisez des doublets.

M. PELLONE. - La portance est engendrée par une répartition de doublets à axes normaux sur le profil plus un tourbillon ponctuel situé au bord de fuite. J'ajouterai que le calcul permet de préciser l'apparition de la cavitation dans les zones calculées expérimentalement.

M. CORNIGLION. - Comment cela se passe-t-il à la naissance de la cavité, à la pointe du profil. Est-ce que vous appliquez la conditon de KUTTA-JOUKOWSKI.

M. PELLONE. - Oui, elle a été appliquée au bord de fuite du profil. La vitesse est déterminée par le calcul.

M. CORNIGLION. - Donc vous appliquez l'égalité des vitesses.
M PELLONE - Nous appliquons la continuité des vitesses aux deux bords de fuite.

M. MICHEL. - En fait, c'est l'égalité

M. le Président. - Y-a-t-il d'autres questions.

M. MICHEL. - Je voudrais faire une remarque par rapport à la communication que nous avons entendue hier et qui comparait les calculs d'aubes avec des figures de cavitation.

Il faut remarquer que cet exposé procède d'une démarche analogue. Les dernières figures donnaient des courbes de début de cavitation en fonction de $C z$ avec une sorte de sceau, de "baquet" de cavitation, comme il a été dit. Les courbes présentées antérieurement étaient obtenues par le calcul, elles sont aussi capables "en gros", de préciser les zones qui vont être les premières sensibles à la cavitation. Il se trouve que cela correspond à peu près aux zones de cavitation qu'indiquait M. LAALI au cours de son exposé.

M. PELLONE. - A l'extrados, le coefficient de pression y est minimal. Du au fait de la discrétisation, on peut commettre des erreurs dans l'évaluation des différences finies. II faudrait faire un calcul beaucoup plus précis que l'approximation adoptée. Cependant on a bien localisé la position des coefficients à l'intrados correspondant au début de la cavitation. Cela confirme les résultats expérimentaux de M. LAALI.

\begin{tabular}{|c|c|}
\hline \multicolumn{2}{|c|}{ Abstract } \\
\hline $\begin{array}{l}\text { Technical progress based on the use of supercavitating } \\
\text { wings with wetted upper surface is expected. An essential } \\
\text { difficulty for the correct use of this type of wing is the } \\
\text { determination of the free field of carvitation. This field } \\
\text { requires knowledge of the pressure coefficients in the region } \\
\text { of the leading edge of the wing; this can be obtained from } \\
\text { experiments or calculation. } \\
\text { With regard to calculation, the fact that the cavity is of } \\
\text { unknown shape, while determination of the pressure coeffi- } \\
\text { cient at the leading edge requires a non-linear procedure, } \\
\text { creates a considerable problem. The calculation method used } \\
\text { is that of discretized particularities. If we assume initially that } \\
\text { the geometry of the cavity is known, we can solve the problem } \\
\text { by distributing a single layer on the wing, a double layer on the } \\
\text { wing, the cavity and the wake, and distributing linear pits } \\
\text { behind the cavity. Solution of the problem leads to non- } \\
\text { uniform pressure in the cavity because of the arbitrary nature } \\
\text { of the geometry chosen to start off the calculation. When the } \\
\text { cavity pressure is made uniform by an iterative procedure, the } \\
\text { length of the cavity can be adjusted in terms of the span of } \\
\text { the wing. We then start again with this shape of cavity, and } \\
\text { the iterations are continued to detail the shape in the three } \\
\text { space dimensions, in order that the slip condition may be } \\
\text { satisfied everywhere on it. } \\
\text { Experimentally, investigations on wings of limited span } \\
\text { require a large installation in order to minimize the influence }\end{array}$ & $\begin{array}{l}\text { of the side walls, the effects of the boundary layer, and the } \\
\text { effects of the housing, as well as the errors arising from the } \\
\text { geometrical definition of the wing itself. The series of tests } \\
\text { reported on here were carried out on a wing of fixed profile } \\
\text { whose sweep-back was varied by rotation around an axis at } \\
\text { the root, and whose aspect ratio was varied by successive } \\
\text { cutting back. The tests were carried out in the TH2-TH8 } \\
\text { hydrodynamic tunnel of the NEYRTEC Company, whose } \\
\text { smallest water-vein permits studies on three-dimensional flows } \\
\text { under acceptable conditions. } \\
\text { The theoretical and experimental results were subjected to } \\
\text { critical analysis of the influence of the principal geometrical } \\
\text { parameters and the area of non-cavitation. In particular, when } \\
\text { then aspect ratio of a ventilated-base wing is reduced, a study } \\
\text { of the slope of the curve, the coefficient of lift in terms of the } \\
\text { angle of attack, and of the spread of non-cavitation reveals a } \\
\text { significant increase in the spread of non-cavitation. This points } \\
\text { to the advantage of using wings of very low aspect ratio in } \\
\text { order to increase the possible incidence deflections of the wing. } \\
\text { Tith regard to the localization of the points where cavitation } \\
\text { appears, for swept-back wings cavitation on the upper surface } \\
\text { appears at about two thirds of the span; on the under surface, } \\
\text { cavitation appears first at the wing root. This result, confirmed } \\
\text { by experience, shows that there may be an advantage in using } \\
\text { a twisted wing, with the a smaller angle for the end sections of } \\
\text { the wing than for those at the root. }\end{array}$ \\
\hline
\end{tabular}

